\title{
TSLC1 inhibits bladder cancer cells proliferation and promotes apoptosis by targeting miR-125b
}

Jun Zhu

Tianjin Medical University General Hospital

Rui Hu

Tianjin Medical University General Hospital

NingJing Ou

Tianjin Medical University General Hospital

Zhen Liang

Tianjin Medical University General Hospital

Wei Zhang

Tianjin Medical University General Hospital

YuXuan Song

Tianjin Medical University General Hospital

Yi Lu

Tianjin Medical University General Hospital JiaQi Kang

Tianjin Medical University General Hospital

\section{YongJiao Yang}

Tianjin Medical University General Hospital

XiaoQiang Liu ( $\sim$ liuxiaoqiangtjykdx@163.com )

\section{Research article}

Keywords: TSLC1,miR-125b,bladder cancer,proliferation,apoptosis

Posted Date: January 29th, 2020

DOI: https://doi.org/10.21203/rs.2.22221/v1

License: (9) This work is licensed under a Creative Commons Attribution 4.0 International License. Read Full License 
TSLC1 inhibits bladder cancer cells proliferation and promotes apoptosis by targeting miR-125b

Jun Zhu ${ }^{1}$,Rui $\mathrm{Hu}^{1}$,NingJing $\mathrm{Ou}^{1}{ }^{1}$ Zhen Liang $^{1}$,Wei Zhang ${ }^{1}$,YuXuan Song ${ }^{1}$,Yi Lu${ }^{1}$, JiaQi Kang ${ }^{1}$,YongJiao Yang $^{1}$,XiaoQiang Liu ${ }^{1}$.

${ }^{1}$ Department of Urology, Tianjin Medical University General Hospital, Tianjin 300052, China

Co-first author:Jun Zhu,Department of Urology,Tianjin Medical University General Hospital,154 Anshan Road, Heping District, Tianjin300052, China.Email: zhujuntjykdx@163.com ;

Rui Hu,Department of Urology,Tianjin Medical University General Hospital,154 Anshan Road, Heping District, Tianjin300052, China.Email: chocolatefree@163.com

Correspondence to: Xiaoqiang Liu, Department of Urology,Tianjin Medical University General Hospital,154 Anshan Road, Heping District, Tianjin300052, China.Email: liuxiaoqiangtjykdx@163.com 


\begin{abstract}
Backgroud: The aim of this study was to investigate the relationship between the expression of tumor suppressor in lung cancer-1 (TSLC1) and miRNA-125b in bladder cancer $(\mathrm{BC})$ pathogenesis.
\end{abstract}

Methods: The expression of miRNA-125b,TSLC1 and p53 in BC cell line was detected by real-time quantitative RT-PCR (RT-qPCR) or western blot. Transwell migration assay was used in the in vitro migration and invison anssay. TSLC1 and p53 expression was evaluated by immunohistochemistric staining in bladder cancer tissues. Results: We showed that the expression of miRNA-125b was significantly decreased in $\mathrm{BC}$ cell line(T24) transfection of miR-125b inhibitor.Knockdown of miRNA-125b promoted the growth and metastasis of T24 cells, while overexpression of miRNA-125b had the opposite effects. Furthermore,TSLC1 was significantly positive correlated with miRNA-125b expression and negative correlated with p53 expression in T24 cells.TSLC1 transfection increased the expression of miRNA-125b,and inhibited BC cell migration and invasion in vitro,and promoted apoptosis. The expression of TSLC1 and p53 was opposite in bladder cancer tissues.

Conclusions: Our data provided strong evidence that TSLC1 inhibited tumorigenesis and development of $\mathrm{BC}$ through up-regulating tumor-suppressive miRNA-125b.

\title{
Keywords
}

TSLC1,miR-125b,bladder cancer,proliferation, apoptosis 


\section{Background}

Bladder cancer(BC) is a malignancy in the world with about 430,000 new cases diagnosed in 2012[1]. Estimated $75 \%$ of newly diagnosed BC are non-muscle invasive, while $70 \%$ of them may undergo tumour relapse and approximately $25 \%$ progress to muscle-invasive[2]. Because of the high incidence, recurrence and progression rate of $\mathrm{BC}$, the five-year survival rate of $\mathrm{BC}$ patients remains terrible. Therefore, illustrating the mechanisms contributing to the development,progression and metastasis of $\mathrm{BC}$ is extremely important.

Tumor suppressor in lung cancer-1 (TSLC1), as a tumor suppressor gene,was first identified in lung carcinoma[3]. TSLC1 encodes a single-pass transmembrane glycoprotein, participates in calcium-independent cell-cell adhesion,and located on human chromosome 11q23[4]. It is now known that TSLC1 is expressed ubiquitously in a variety of human tissues, such as lung, brain, testes as well as most epithelial and neuronal tissues[5]. Recently, TSLC1 expression was found to be lost or downregulated in specimens and cell line derived from many types of human tumors, including lung, breast, nasopharyngeal, cervical, liver and colon cancer, meningioma,cutaneous melanoma and T-cell leukemia/lymphoma, and the lack of TSLC1 expression was associated with the occurrence and development of these cancers[6-10]. But so far,the biological functions of TSLC1 are still unclear. [11].

MicroRNAs (miRNAs) are small non-coding RNAs of about 22 nucleotides in length[12]. They are aberrantly expressed in cancers,play important regulatory roles as oncogenes or tumor-suppressor genes and be involved in many cellular processes, 
such as tumorigenesis[13-15].Specifically, based on the results of microRNA microarray in some cancer tissues, microRN-125b(miR-125b) is downregulated in malignancies such as ovarian cancer, bladder cancer and breast cancer but is upregulated in leukemia,prostate cancer and glioma[16-17].Interestingly, most published studies have focused on analyzing the effects of miR-125b on cell proliferation. Overexpression of miR-125b suppresses the proliferation of bladder cancer cells and inhibits apoptosis in prostate cancer cells, and regulates the expression of $\mathrm{p} 53[18-20]$. However, the function of $\mathrm{miR}-125 \mathrm{~b}$ and the molecular mechanisms in bladder cancer metastasis are still unclear.

In our analysis, we frst investigated the levels of TSLC1 and miR-125b in BC cell line. We found that both of them were downregulated in $\mathrm{BC}$ cells, and lower miR-125b expression levels were strongly associated with BC cell proliferation, migration and invasion regulated by TSLC1. TSLC1 may target miR-125b and promot the miR-125-induced inhibition of the tumorigenic properties of $\mathrm{BC}$ cells.

\section{Methods}

Cell culture and Cell transfection assays

The human bladder cancer cell line T24 was donated by Tianjin Institute of Urology, Tianjin, China. The T24 cells were cultured in RPMI-1640 medium supplemented with $10 \%$ fetal bovine serum (FBS,Gibco, USA), $100 \mathrm{U} / \mathrm{ml}$ penicillin, and $100 \mu \mathrm{g} / \mathrm{ml}$ streptomycin at $37^{\circ} \mathrm{C}$ with $5 \% \mathrm{CO} 2$.

The oligonucleotides of miR-125b inhibitor and miRNA inhibitor control were 
purchased from Aidlab (Aidlab,Beijing,China). The TSLC1 for transfection was also purchased from Aidlab (Aidlab,Beijing,China). The cell transfection assays were carried out by using Lipofectamine 2000 reagent (Invitrogen) according to the manufacturer's protocol.Lentivirus productions that downexpressed miR-125b (LV-miR-125b) and expressed GFP alone (LV-control) were purchased from Aidlab (Aidlab,Beijing,China). T24 cells were infected with the productions plus $5 \mu \mathrm{g} / \mathrm{mL}$ polybrene (Sigma-Aldrich, St. Louis, USA) according to the manufacturer's instructions. The GFP+ cells accounted for over $90 \%$ of infected cells, as determined by fluorescence-activated cell sorting (FACS) analysis.

RNA extraction and quantitative real-time PCR

RNA from cultured cells were extracted using the TRIzol reagent (Aidlab,Beijing,China) according to the manufacturer's methods. Reverse transcription was performed with $1 \mu \mathrm{g}$ RNA using the First Stand cDNA Synthesis Kit (TIANGEN Biotechnology,Beijing,China). Real-time PCR analysis was carried out with the SYBR Green PCR Master Mix (ABI) with the following primers: miR-125b, forward 5'-TGCGCTCCCTGAGACCCTAACT and reverse 5'-CCAGTGCAGGGTCCGAGGTATT; U6 RNA, forward 5'-CGCTTCGGCAGCACATATAC and reverse 5'-AAATATGGAACGCTTCACGA was used as the international control.TSLC1, forward 5'-GGTGTTCGCCATGCTGTGCTTGCTC and reverse 5'-CGTCTGCTGCGTCATCGGCTCCTTT. $\beta$-actin RN,forward 
5'-AGCGAGCATCCCCCAAAGTT and reverse 5'-GGGCACGAAGGCTCATCATT was used as the international control. The relative expression of miR-125b was normalized to the expression of U6 RNA and TSLC1 was normalized to $\beta$-actin RNA with the 2- $\Delta \Delta \mathrm{CT}$ method.All qRT-PCR reactions were performed in triplicate.

\section{Western blot analysis}

Western blot analysis was performed according to standard procedures. Total protein extracts from each group of cells were resolved by $10 \%$ SDS-PAGE and transferred onto polyvinylidene fluoride (PVDF) (Millipore) membranes. After blocking, the PVDF membranes were washed 3 times for $10 \mathrm{~min}$ with tris-buffered saline with Tween 20 (TBST) at room temperature and incubated with primary antibodies: p53 (1:2000; Wuhan, China), $\beta$-actin (1:200; Wuhan, China). Following extensive washing, membranes were incubated with secondary antibody IgG (1:5000; Wuhan, China) for $1 \mathrm{~h}$. After washing again 3 times for 10 min with TBST at room temperature, the immunoreactivity was visualized by enhanced chemiluminescence (ECL kit,Thermo,USA).

Cell migration and invasion assays

Transwell chambers (Corning Incorporated,Corning, NY, USA) were used in the in vitro migration and invasion assays. For migration assays, $2 \times 10^{5}$ cells were resuspended in the top chamber with the noncoated membrane (24-well insert; pore size, $8 \mu \mathrm{m}$;Corning). For invasion assays, $2 \times 10^{5}$ cells were plated in the top chamber 
with Matrigel-coated membrane (24-well insert; pore size, $8 \mu \mathrm{m}$; Corning). In both assays, cells were plated in medium without serum, and medium $10 \%$ FBS in the lower chamber was used as a chemoattractant. After incubation for $24 \mathrm{~h}$, the cells that did not migrate or invade through the pores were removed by a cotton swab. Cells on the lower surface of the membrane were fixed with $4 \%$ paraformaldehyde and stained with $0.1 \%$ crystal violet and counted. Each experiment was performed in triplicate.

Immunofluorescent staining for TSLC1 and p53

10 bladder cancer tissue samples were obtained from patients with $\mathrm{BC}$ who underwent surgery in Tianjin Medical University General Hospital from 2018-2019. TSLC1 and p53 expression was evaluated by immunohistochemistric staining. Briefly, after 5-um sections were deparaffinized, antigen retrieval was performed by use of heat-induced epitoperetrieval with $10 \mathrm{mM}$ citrate buffer. Sections were incubated with a monoclonal antibody against TSLC1 (Wuhan, China) at 1:300 dilution and p53 (Wuhan, China) at 1:500 dilution. The TSLC1 and p53 antibodies were detected using the avidinbiotin-peroxidase technique (Dako REAL EnVision Detection System, Dako). The expression levels of TSLC1 and p53 were determined by a pathologist.

\section{Statistical analysis}

Data were presented as mean \pm SEM unless otherwise indicated. Student's $t$ test was used for statistical analysis, with $\mathrm{P}<0.05$ considered significant. 


\section{Results}

Silencing miR-125b positively regulates bladder cancer cell migration and invasion in vitro.

To investigate the function of miR-125b in bladder cancer cell migration and invasion, miR-125b inhibitor and inhibitor control were transiently transfected into T24 cells, and the downexpression of miR-125b was confirmed by qRT-PCR in T24 cells and T24-GFP cells. As shown in Fig. 1, the qPCR analysis indicated that the abundance of miR-125b was signifcantly decreased in T24-miR-125b inhibitor cells(T24-miR-125b-) comparison with that of T24 cells and T24-GFP cells. Down-regulation of miR-125b significantly promoted the migratory and invasive abilities of T24 cells compared with T24 cells and T24-GFP cells(Fig. 2,3). These data confirm that miR-125b exert a significant role in $\mathrm{BC}$ migration and invasion.

Transfection of TSLC1 inhibites the expression of miR-125b in vitro.

To verify whether TSLC1 target the expression of miR-125b, TSLC1 was transfected into T24 cells and T24-miR-125b inhibitor cells. The transfection efficiency and the abundance of miR-125b and TSLC1 was also detected by qRT-PCR . The expression of TSLC1 was signifcantly increased in T24-TSLC1 cells and T24-miR-125b--TSLC1 cells comparison with that of T24 cells and T24-GFP cells(Fig. 4A,4B). While compared with T24-miR-125b-cells, the expression of miR-125b was signifcantly increased in T24-miR-125b--TSLC1 cells(Fig. 4C).These findings suggest that TSLC1 play a vital role in the expression of miR-125b.

Transfection of TSLC1 inhibites bladder cancer cell migration and invasion 


\section{in vitro.}

To further confirm the role of TSLC1 in bladder cancer cell migration and invasion,migratory and invasive abilities of T24 cells were also evaluated.We found that overexpression of TSLC1 suppressed the migration and invasion of T24 cells (Fig. 5,6). These results suggest that TSLC1 might play a role in $\mathrm{BC}$ tumorigenesis by influencing the expression of miR-125b.

p53 is involved in the migration and invasion regulated by TSLC1 or miR-125b in bladder cancer.

As far as we know, the role of p53 in bladder cancer have not been illuminated. To investigate the relationship between p53 and TSLC1, the abundance of p53 were detected by qRT-PCR and Western blot in T24 cells,T24-GFP cells,T24-miR-125b-cells and T24-miR-125b--TSLC1 cells.Besides,immunofluorescent staining was also used.We found that the abundance of p53 was signifcantly increased in T24-miR-125b-cells compared with T24 cells and T24-GFP cells,but decreased in T24-miR-125b--TSLC1 cells in comparison with T24-miR-125b-cells(Fig. 7). And the abundance of p53 and TSLC1 in bladder cancer tissues was consistent with the upper results(Fig. 8). These findings suggest that p53 maybe a functional target of TSLC1 or miR-125b.

\section{Discussion}

Bladder cancer is one of the five most common malignancies allover the world,and it is the 2nd most common cause of death in patients with genitourinary 
tract malignancies[21-22] . BC has strong potential for invasion and metastasis, which always results in poor prognosis and a 5-year survival rate of less than 50\% [23]. Therefore, it is urgent to explore the tumorigenesis of $\mathrm{BC}$ and to find reliable biomarkers and effective therapeutic targets for improving the survival rate of patients with BC.

TSLC1 could suppress tumor growth and loss of TSLC1 function could lead to invasion or metastasis of cancer cells[24]. Loss or low expression of tumor suppressor gene TSLC1 has been found in specimens and cell lines derived from many types of human tumors; and the lack of TSLC1 expression was associated with the occurrence and development of these cancers. The genetic or epigenetic aberrations affecting TSLC1 have been documented in tumor tissues of a variety of origins, including the esophagus, stomach, liver and pancreatic cancers as well as other digestive tumors, laryngeal cancer, canine meningiomas, breast cancer nasopharyngeal cancer, hepatocellular carcinoma, lung cancer adult acute lymphoblastic cell leukemia, and colon cancer [25-26]. miR-125b was implicated to have close relationship with cell proliferation and differentiation.Recent studies have shown that,overexpression of miR-125b plays an important role in prostate cancer, pancreatic cancer, and oligodendroglial cancer [27].Inversely, miR-125b is down-regulated in cancers such as ovarian cancer,bladder cancer, and liver cancer [28-29].Together with our results, these data suggest that miR-125b is likely to play a crucial role in bladder cancer. However, the biological function of TSLC1 and miR-125b in bladder tumorigenesis is still unknown. Our results demonstrated that both of TSLC1 and miR-125b were 
involved in tumorigenesis of bladder cancer.

In this study, we have identified that the expression of miR-125b was significantly decreased in bladder cancer cell line with transfection of miR-125b inhibitor.Importantly,silencing of miR-125b could promote migration and invasion proliferation of bladder cancer cells in vitro. Furthermore,transfection of TSLC1 following miR-125b inhibition could improve the expression of miR-125b, and have the converse effects on bladder cancer cells. This is the first study to investigate the role of TSLC1 in bladder cancer cell line, and also the first study to identify that miR-125b is a direct and functional target of TSLC1.Moreover,we also have identified that the expression of p53 was significantly increased in bladder cancer cell line with transfection of miR-125b inhibitor.Conversely,it was significantly decreased by transfection of TSLC1 following miR-125b inhibition.However, we did not further explore whether p53 was the target molecule of miR-125b in $\mathrm{BC}$, and these parts will further explore in subsequent studies.

In summary, our study demonstrates that both of TSLC1 and miR-125b have a tumor suppressive function in human bladder cancer.And miR-125b,an important miRNA for migration and invasion of bladder cancer cells, is identified as a target of TSLC1. Besides,p53maybe a a target of miR-125b. We believe that TSLC1 and miR-125b might have a potential role in gene therapy and may be a promising candidate biomarker for diagnosis of human bladder cancer.

\section{Conclusions}


This study investigated the relationship between TSLC1 and miRNA-125b in BC.It provided strong evidence that TSLC1 and miRNA-125b contributed to a negative prognosis in BC.TSLC1 could inhibit tumorigenesis and development of BC through up-regulating tumor-suppressive miRNA-125b.

\section{List of abbreviations}

TSLC1:tumor suppressor in lung cancer-1

BC:bladder cancer

RT-qPCR:real-time quantitative RT-PCR

\section{Declarations}

- Ethics approval and consent to participate

The study was approved and supervised by the Ethics Committee of Tianjin Medical University General Hospital.All patients provided written informed consent prior to operation, and they all know their bladder cancer tissue samples maybe used in the experiment.

- Consent for publication

Not applicable.

- Availability of data and materials

All data generated or analysed during this study are included in this published 
article.And the datasets used and/or analysed during the current study are available from the corresponding author on reasonable request.

- Competing interests

The authors declare that they have no competing interests.

- Funding

This research is supported by the Natural Science Foundation of Tianjin City (grant No. 16JCZDJC34600). The funding body had no role in the study design, data collection, data interpretation, or writing of this manuscript.

- Authors' contributions

$\mathrm{JZ}$ and RH contributed equally to this work.RH drafted the manuscript.JZ revised the manuscript.WZ,ZL,NJO,YXS,Y L,JQK,and YJY performed the experiments and analyzed the data.XQL supervised the research. All authors read and approved the final manuscript.

- Acknowledgements

Not applicable.

\section{References}

1. Antoni S, Ferlay J, Soerjomataram I,et al.Bladder Cancer Incidence and Mortality: A Global Overview and Recent Trends. Eur Urol. 2017;71:96-108.

2. Ooms EC, Anderson WA, Alons CL,et al.Analysis of the performance of pathologists in the grading of bladder tumors. Hum Pathol. 1983;14:140-43. 
3. Murakami Y, Nobukuni T, Tamura K,et al. Localization of tumor suppressor activity important in nonsmall cell lung carcinoma on chromosome 11q. Proc Natl Acad Sci USA. 1998;95:8153-58.

4. Chen YG, Yang YJ, Liu L,et al.Tumor suppressor in lung cancer-1 (TSLC1) is a prognostic predictor for the recurrence and progression of non-muscle-invasive bladder cancer.Urol Int. 2016;96:142-47.

5. Liang QL, Chen GQ, Li ZY, et al. Function and histopathology of a cell adhesion molecule TSLC1 in cancer. Cancer Invest. 2011;29:107-12.

6. Zhang J, Ning J, Geng J, et al. Down-regulation of tumor suppressor in lung cancer 1 (TSLC1) expression correlates with poor prognosis in patients with colon cancer.J Mol Histol. 2012;43:715-21.

7. Nakahata S, Saito Y, Marutsuka $\mathrm{K}$, et al. Clinical significance of CADM1/TSLC1/IgSF4 expression in adult T-cell leukemia/lymphoma.Leukemia. 2012;26:1238-46.

8. You Y, Wang SH, Zhang JF, et al. TSLC1 expression discriminates cutaneous melanomas from dysplastic nevi. Melanoma Res. 2012;22:430-35.

9. Liang QL, Wang BR, Li ZY,et al. Construction of eukaryotic expression vector of TSLC1 gene. Arch Med Sci. 2011;7:579-85.

10. Lu B, Di W, Wang H, et al.Tumor suppressor TSLC1 is implicated in cell proliferation, invasion and apoptosis in laryngeal squamous cell carcinoma by regulating Akt signaling pathway. Tumour Biol. 2012; 33:2007-17.

11. Liang QL, Chen GQ, Liu QL,et al. Tumor suppressor TSLC1 inhibits growth, 
proliferation, invasiveness and angiogenesis in nude mice xenografted tumor of Eca109 cells.Int J Clin Exp Med. 2014;7:1507-15.

12. Bartel DP.MicroRNAs: Genomics, biogenesis, mechanism, and function. Cell. 2004;116:281-97.

13. Cui X, Kong C, Zhu Y,et al. miR-130b, an onco-miRNA in bladder cancer, is directly regulated by $\mathrm{NF}-\kappa \mathrm{B}$ and sustains $\mathrm{NF}-\mathrm{\kappa B}$ activation by decreasing Cylindromatosis expression.Oncotarget. 2016;7:48547-61.

14. Song T, Zhang X, Yang G,et al. Decrement of miR-199a-5p contributes to the tumorigenesis of bladder urothelial carcinoma by regulating MLK3/NF- $\mathrm{BB}$ pathway. Am J Transl Res. 2015;7: 2786-94.

15. Lee JY, Ryu DS, Kim WJ,et al. Aberrantly expressed microRNAs in the context of bladder tumorigenesis. Investig Clin Urol. 2016; 57: S52-9.

16. Ichimi T, Enokida H, Okuno Y,et al. Identification of novel microRNA targets based on microRNA signatures in bladder cancer. Int J Cancer. 2009;125: 345-52.

17. Iorio MV, Ferracin M, Liu CG,et al. MicroRNA gene expression deregulation in human breast cancer. Cancer Res. 2005; 65:7065-70.

18. Huang L, Luo J, Cai Q,et al. MicroRNA-125b suppresses the development of bladder cancer by targeting E2F3. Int J Cancer. 2011; 128:1758-69.

19. Shi XB, Xue L, Ma AH, et al. miR-125b promotes growth of prostate cancer xenograft tumor through targeting pro-apoptotic genes.Prostate. 2011;71: 538-49.

20. Le MT, Teh C, Shyh-Chang N,et al. MicroRNA-125b is a novel negative regulator of p53. Genes Dev. 2009; 23: 862-76. 
21. Jemal A, Siegel R, Ward E,et al. Cancer statistics. CA Cancer J Clin. 2008;58:71-96.

22. Parkin DM, Bray F, Ferlay J,et al. Global cancer statistics. CA Cancer J Clin. 2005;55:74-108.

23. Lu B, Di W, Wang H,et al. Tumor suppressor TSLC1 is implicated in cell proliferation, invasion and apoptosis in laryngeal squamous cell carcinoma by regulating Akt signaling pathway. Tumour Biol. 2012; 33:2007-17.

24. Zhang J, Ning J, Geng J, et al.Down-regulation of tumor suppressor in lung cancer 1 (TSLC1) expression correlates with poor prognosis in patients with colon cancer. J Mol Histol. 2012; 43: 715-21.

25. Mark B, Frankel WL, Fabio P, et al. MicroRNA expression patterns to differentiate pancreatic adenocarcinoma from normal pancreas and chronic pancreatitis.JAMA. 2007;297:1901-8.

26. Nelson PT, Baldwin DA, Kloosterman WS,et al. RAKE and LNA-ISH reveal microRNA expression and localization in archival human brain. Rna Publ Rna Soc. 2006;12:187-91.

27. Guan Y, Yao H, Zheng Z,et al.MiR-125b targets BCL3 and suppresses ovarian cancer proliferation. Int J Cancer. 2011;128:2274-83.

28. Huang L, Luo J, Cai Q,et al. MicroRNA-125b suppresses the development of bladder cancer by targeting E2F3. Int J Cancer. 2011;128:1758-69.

29. Liang L, Wong CM, Ying Q,et al. MicroRNA-125b suppressed human liver cancer cell proliferation and metastasis by directly targeting oncogene 
LIN28B2.Hepatology. 2010;52:1731-40.

Figure Legends

Fig. 1 miR-125b expression in T24 cells,T24-GFP cells and T24-miR-125b- cells. miR-125b mRNA expression in T24 cells,T24-GFP cells and T24-miR-125bcells(transfected with miR-125b inhibitor) were measured by qRT-PCR.U6 was used as a reference gene for qRT-PCR. (**,P $<0.01$ vs. T24 cells).

Fig. 2 miR-125b knockdown promoted bladder cancer cell invasion in vitro. Invasion assay of T24 cells(2A), T24-GFP cells(2B) and T24-miR-125b-cells(2C). Invasion: $\times 200$. (**,P $<0.01$ vs. T24 cells, 2D).Each experiment was performed in triplicate.

Fig. 3 miR-125b knockdown promoted bladder cancer cell migration in vitro. Migration assay of T24 cells(3A), T24-GFP cells(3B) and T24-miR-125b- cells(3C). Migration: $\times 200 .(* *, P<0.01$ vs. T24 cells, 3D).Each experiment was performed in triplicate.

Fig. 4 TSLC1 expression and TSLC1 promoted miR-125b expression in vitro. (4A)TSLC1 mRNA expressions in T24 cells,T24-GFP cells and T24-TSLC1 cells(transfected with TSLC1) were measured by qRT-PCR.U6 was used as a reference gene for qRT-PCR. (**,P $<0.01$ vs. T24 cells). 
(4B)TSLC1 mRNA expressions in T24 cells,T24-GFP cells, T24-miR-125b-cells and T24-miR-125b--TSLC1 cells were measured by qRT-PCR. U6 was used as a reference gene for qRT-PCR. (**,P $<0.01$ vs. T24-miR-125b- cells).

(4C)miR-125b mRNA expressions in T24 cells,T24-GFP cells,T24-miR-125b-cells and T24-miR-125b--TSLC1 cells were measured by qRT-PCR. U6 was used as a reference gene for qRT-PCR. (**,P $<0.01$ vs. T24-miR-125b- cells).

Fig. 5 TSLC1 negatively regulates cell invasion in vitro. Invasion assay of T24 cells(5A), T24-GFP cells(5B) and T24-TSLC1+miR-125b inhabitor cells(infected with TSLC1-overexpressing lentivirus)(5C). Invasion: $\times 200$. (**,P<0.01 vs. T24 cells,5D). Each experiment was performed in triplicate.

Fig. 6 TSLC1 negatively regulates cell migration in vitro. Migration assay of T24 cells(6A), T24-GFP cells(6B) and T24-TSLC1+miR-125b inhabitor cells(6C).Migration: $\times 200 . \quad(* *, P<0.01$ vs. T24 cells,6D). Each experiment was performed in triplicate.

Fig. 7 p53 protein and mRNA expression in T24 cells, T24-GFP cells,T24-miR-125b-cells and T24-miR-125b--TSLC1 cells.p53 proteins were measured by Western blot (7A and 7B). $\beta$-actin was used as a loading control for Western blot. p53 mRNA expressions were measured by qRT-PCR(7C). U6 was used as a reference gene for qRT-PCR.( ${ }^{* *}, \mathrm{P}<0.01$ vs. T24-miR-125b- cells). 
Fig. 8 Immunohistochemistry of TSLC1 and p53. TSLC1 expression in bladder cancer tissues.a: $\times 100(8 \mathrm{~A}), \mathrm{b}: \times 400(8 \mathrm{~B})$. p53 expression in bladder cancer tissues.c: $\times 100(8 \mathrm{C}), \mathrm{d}: \times 400(8 \mathrm{D})$. 
Figures

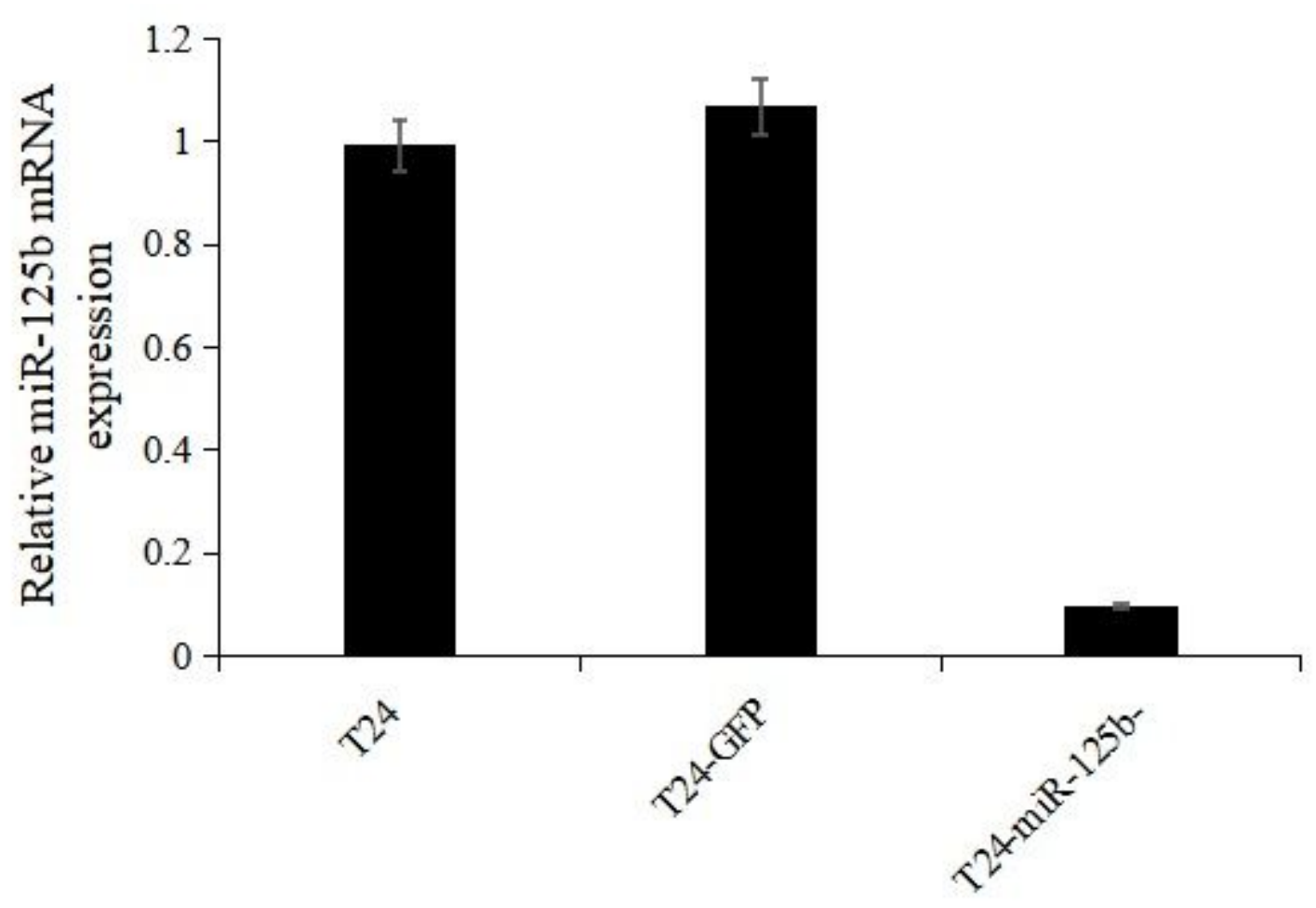

Figure 1

miR-125b expression in T24 cells,T24-GFP cells and T24-miR-125b- cells. miR-125b mRNA expression in T24 cells,T24-GFP cells and T24-miR-125bcells( transfected with miR-125b inhibitor) were measured by qRT-PCR.U6 was used as a reference gene for qRT-PCR. (**,P $<0.01$ vs. T24 cells). 

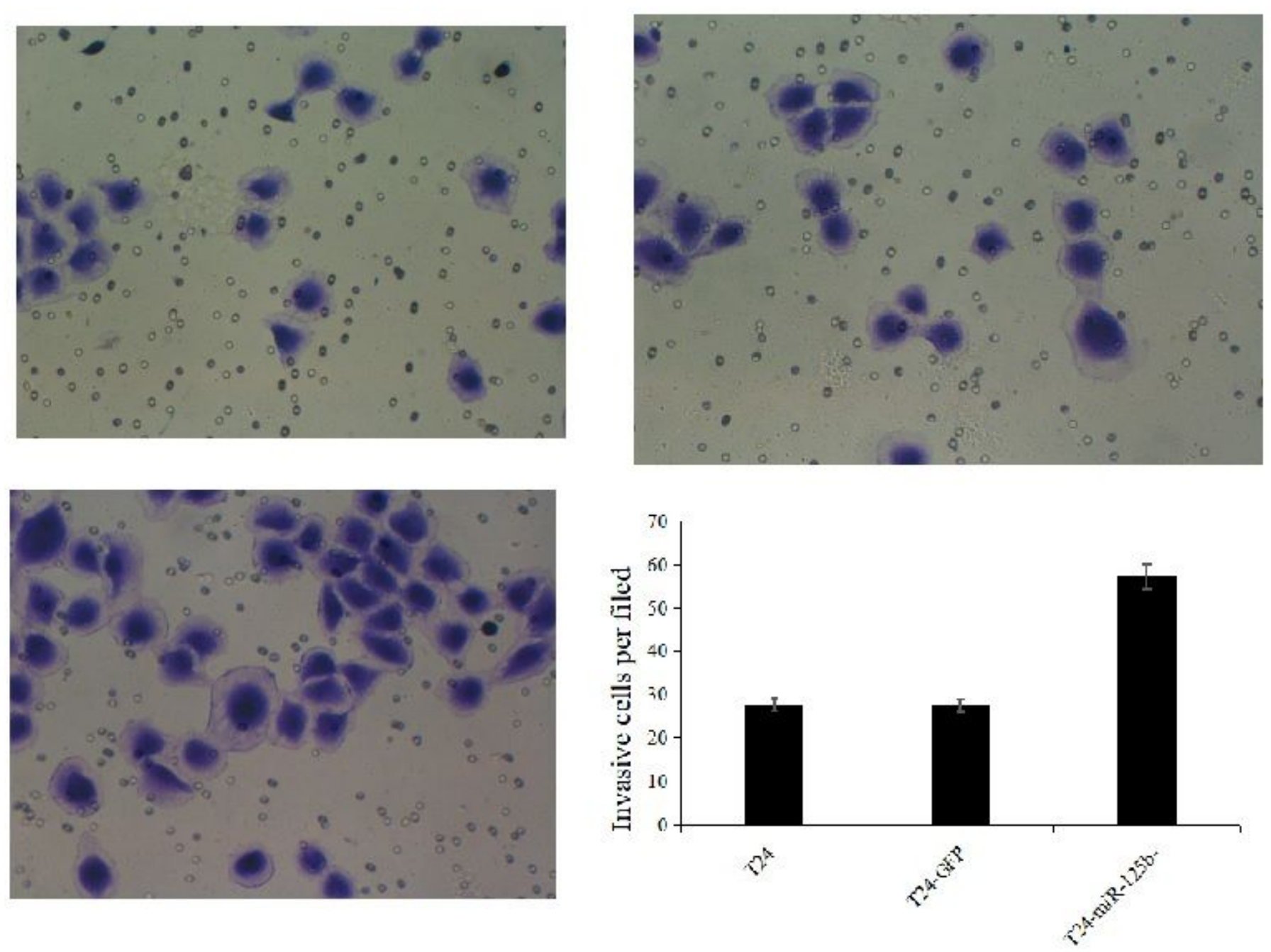

Figure 2

miR-125b knockdown promoted bladder cancer cell invasion in vitro. Invasion assay of T24 cells(2A), T24-GFP cells(2B) and T24-miR-125b- cells(2C). Invasion: $\times 200$. ( $* \star, P<0.01$ vs. T24 cells, 2D). Each experiment was performed in triplicate. 

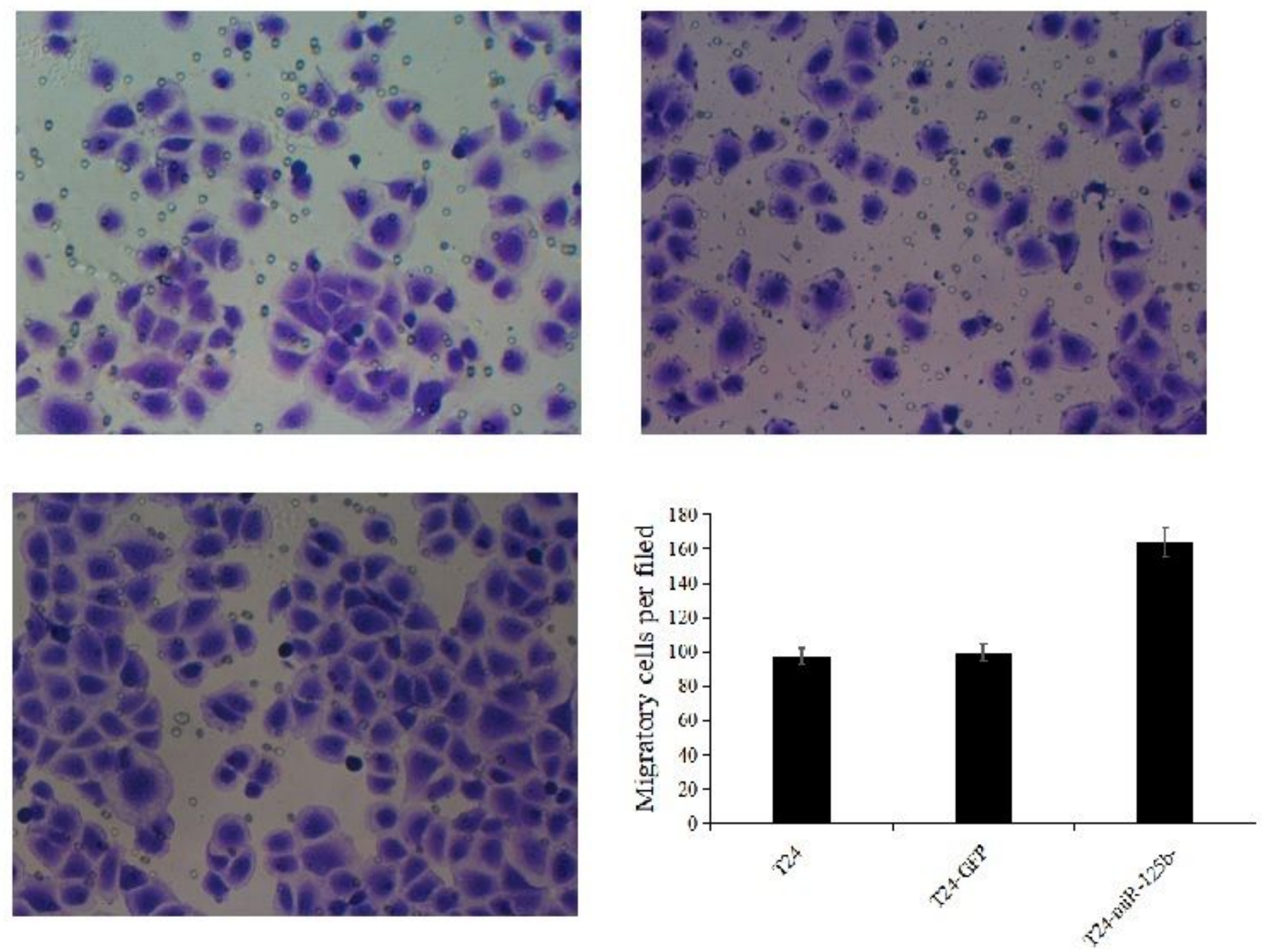

Figure 3

miR-125b knockdown promoted bladder cancer cell migration in vitro. Migration assay of T24 cells(3A), T24-GFP cells(3B) and T24-miR-125b- cells(3C). Migration: $\times 200$. (**,P $<0.01$ vs. T24 cells, 3D).Each experiment was performed in triplicate. 


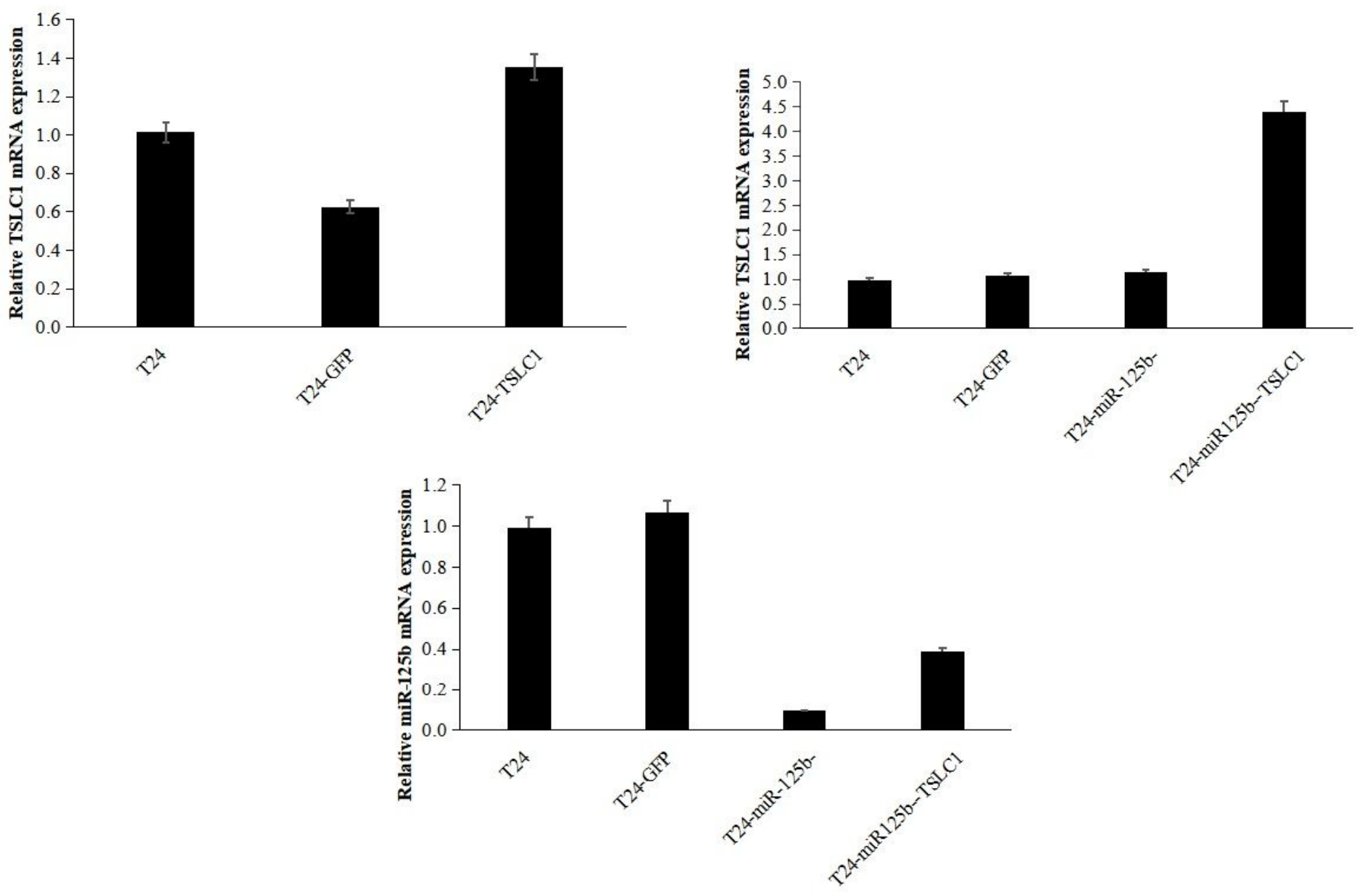

Figure 4

4 TSLC1 expression and TSLC1 promoted miR-125b expression in vitro. (4A)TSLC1 mRNA expressions in T24 cells,T24-GFP cells and T24-TSLC1 cells(transfected with TSLC1) were measured by qRT-PCR.U6 was used as a reference gene for qRT-PCR. ( $\star \star, P<0.01$ vs. T24 cells). (4B)TSLC1 mRNA expressions in T24 cells,T24-GFP cells,T24-miR-125b-cells and T24-miR-125b-TSLC1 cells were measured by qRT-PCR. U6 was used as a reference gene for qRT-PCR. (**,P $<0.01$ vs. T24-miR-125b-cells). (4C)miR-125b mRNA expressions in T24 cells,T24-GFP cells,T24-miR-125b-cells and T24-miR-125b-TSLC1 cells were measured by qRT-PCR. U6 was used as a reference gene for qRT-PCR. ( ${ }^{\star \star}, P<0.01$ vs. T24-miR-125bcells) 

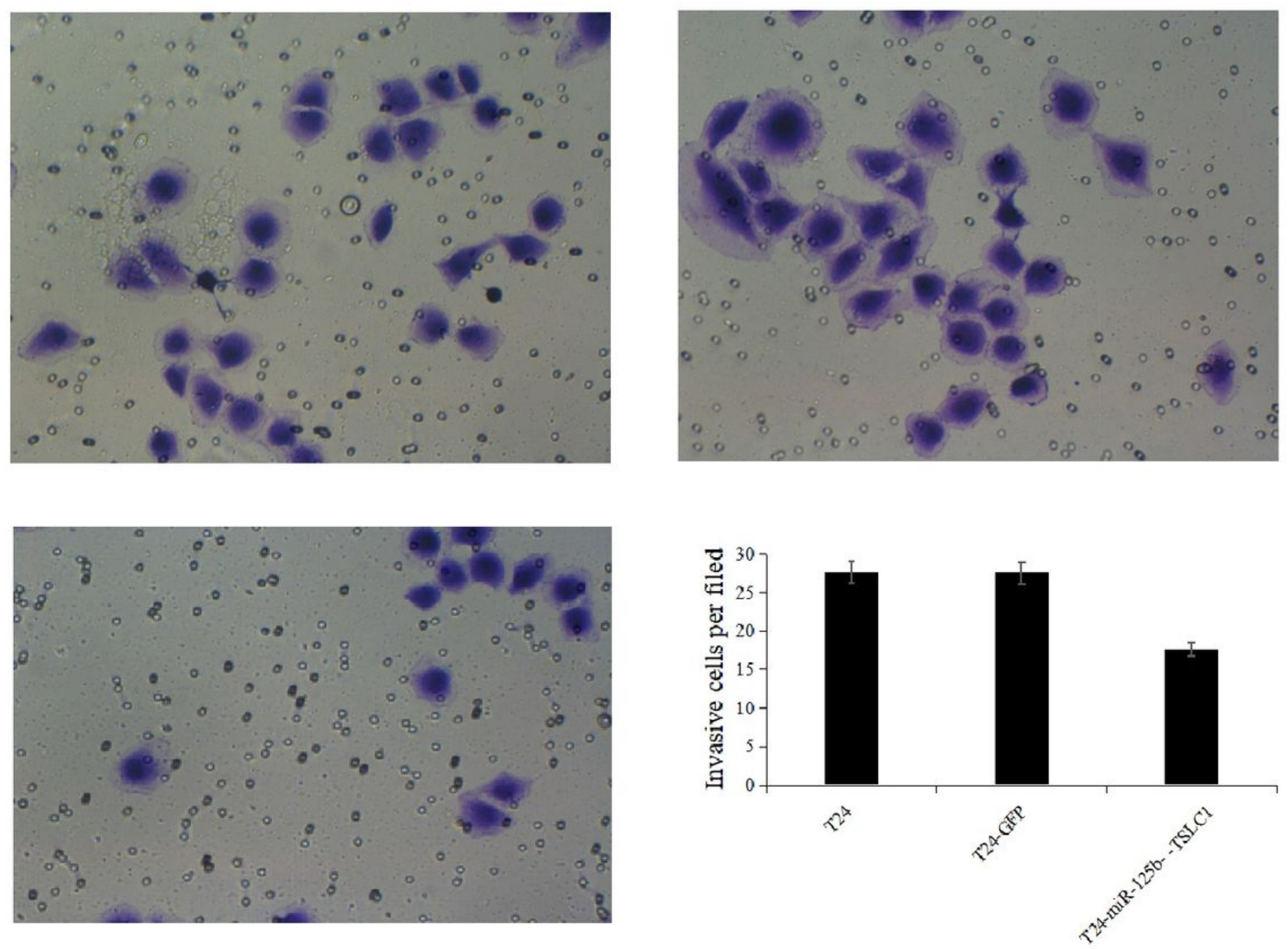

Figure 5

TSLC1 negatively regulates cell invasion in vitro. Invasion assay of T24 cells(5A), T24-GFP cells $(5 B)$ and T24-TSLC1+miR-125b inhabitor cells(infected with TSLC1-overexpressing lentivirus)(5C). Invasion: $\times 200$. ( $*$,$P<0.01$ vs. T24 cells,5D). Each experiment was performed in triplicate. 

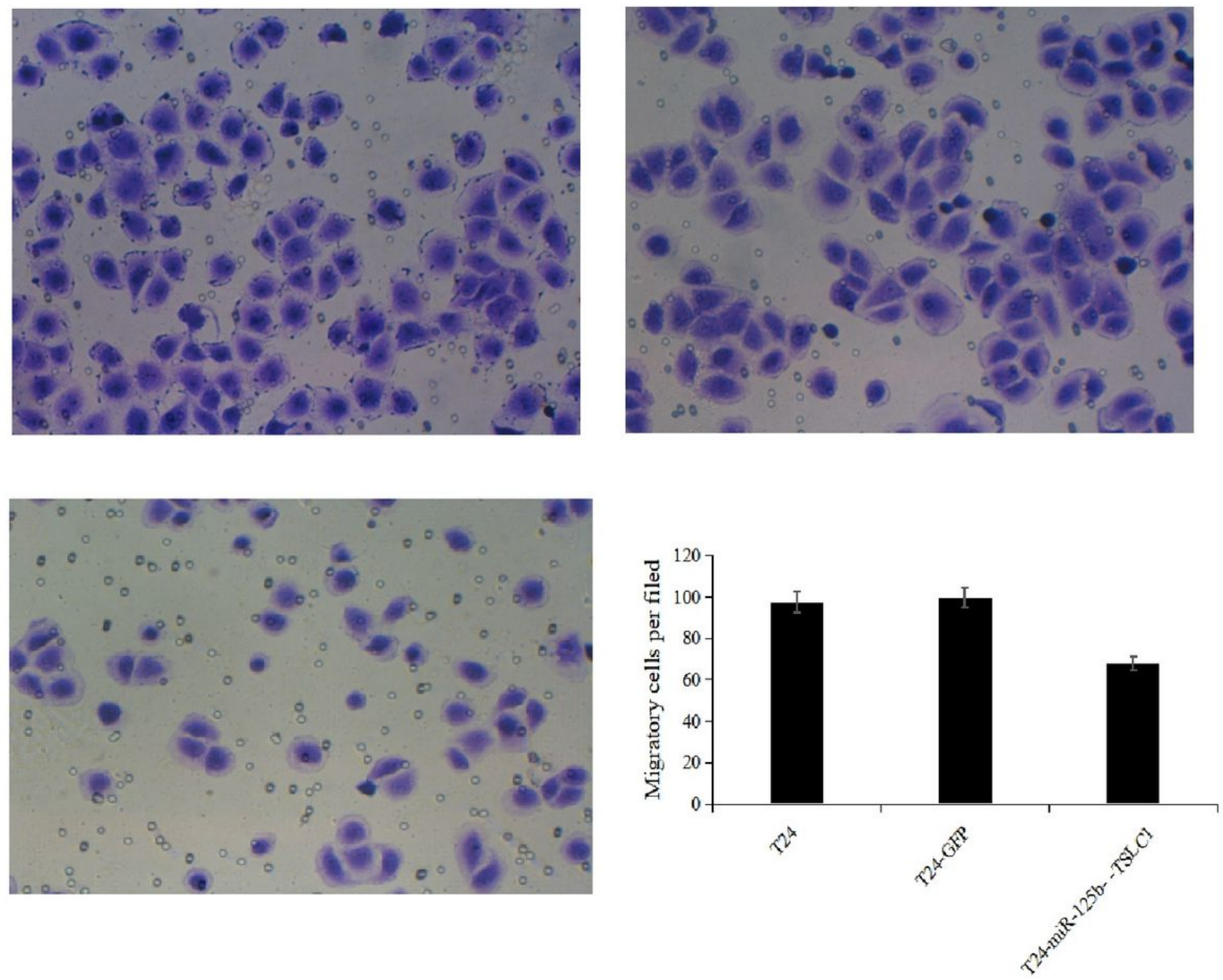

Figure 6

TSLC1 negatively regulates cell migration in vitro. Migration assay of T24 cells(6A), T24-GFP cells(6B) and T24-TSLC1+miR-125b inhabitor cells(6C).Migration: $\times 200$. ( ${ }^{*}, P<0.01$ vs. T24 cells,6D). Each experiment was performed in triplicate. 


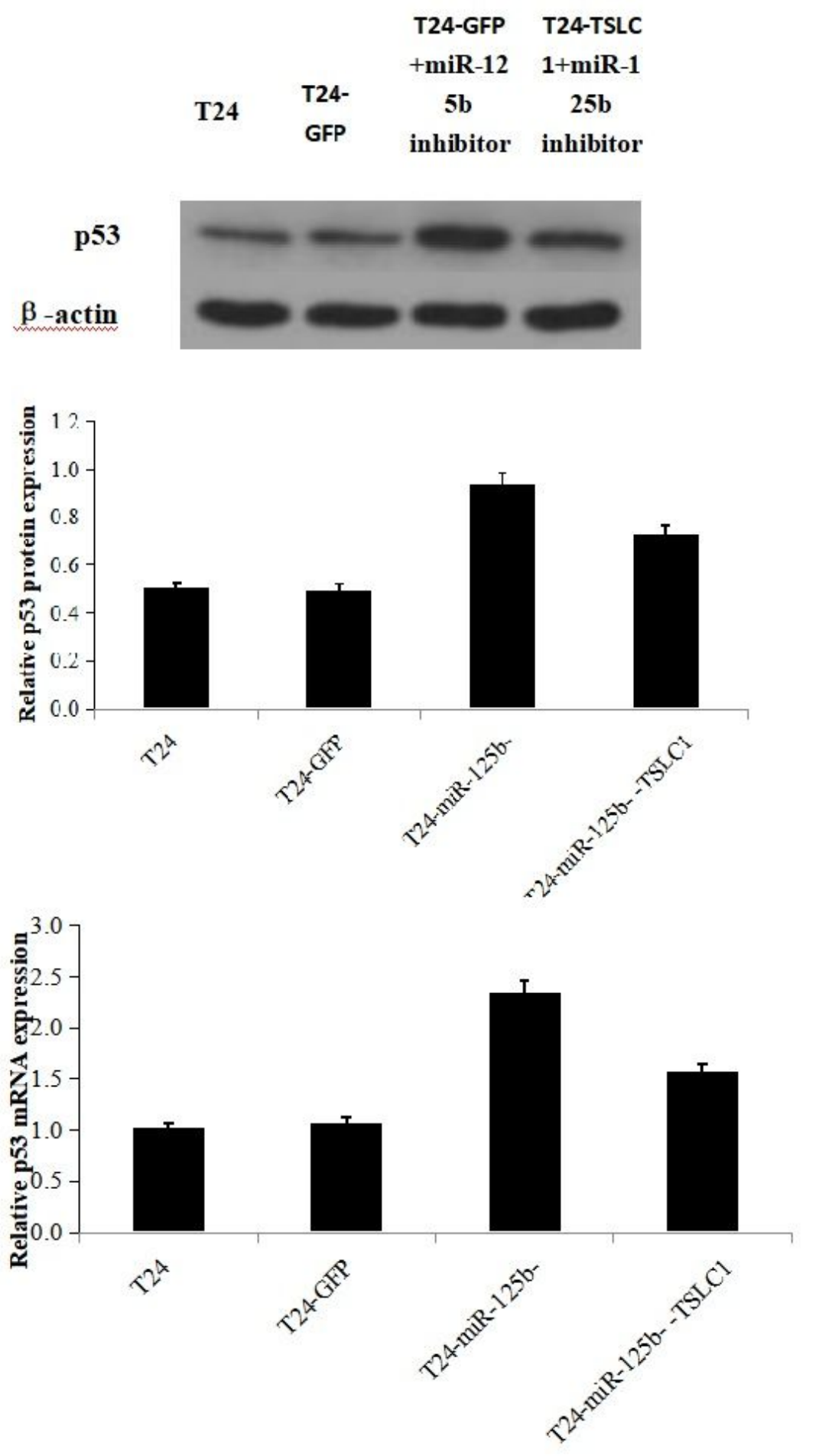

Figure 7

p53 protein and mRNA expression in T24 cells,T24-GFP cells,T24-miR-125b-cells and T24-miR-125b-TSLC1 cells.p53 proteins were measured by Western blot (7A and 7B). $\beta$-actin was used as a loading control for Western blot. p53 mRNA expressions were measured by qRT-PCR(7C). U6 was used as a reference gene for qRT-PCR.( ${ }^{\star *}, P<0.01$ vs. T24-miR-125b- cells). 

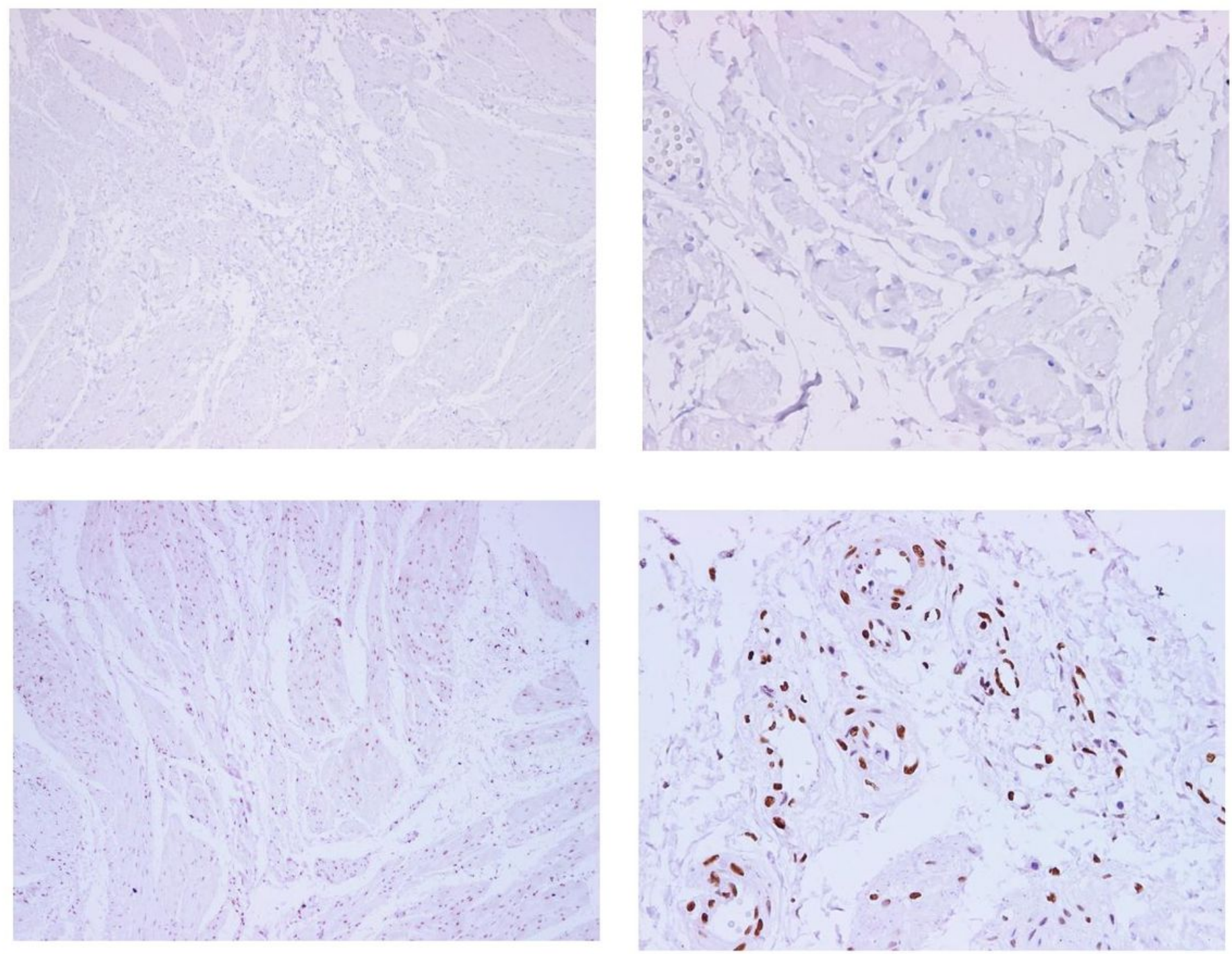

Figure 8

Immunohistochemistry of TSLC1 and p53. TSLC1 expression in bladder cancer tissues.a: $\times 100(8 \mathrm{~A}), \mathrm{b}$ : $\times 400(8 B)$. p53 expression in bladder cancer tissues.c: $\times 100(8 C), d: \times 400(8 D)$. 